\title{
On the Quantization of the Abelian Chern-Simons Coefficient at Finite Temperature
}

\author{
N. Bralić* \\ Facultad de Física, Pontificia Universidad Católica de Chile \\ Casilla 306, Santiago 22, Chile \\ C.D. Fosco ${ }^{\dagger}$ \\ International Centre for Theoretical Physics \\ P.O. Box 586, 34100 Trieste, Italy \\ F.A. Schaposnik ${ }^{\ddagger}$ \\ Departamento de Física, Universidad Nacional de La Plata \\ C.C. 67, (1900) La Plata, Argentina
}

\begin{abstract}
We show that when the Abelian Chern-Simons theory coupled to matter fields is quantized in a vacuum with non vanishing magnetic flux (or electric charge), the requirement of gauge invariance at finite temperature leads to the quantization of the Chern-Simons coefficient and its quantum corrections, in a manner similar to the non-Abelian case.
\end{abstract}

\footnotetext{
*nbralic@lascar.puc.cl

$\dagger$ fosco@ictp.trieste.it

†nvestigador CICBA
} 
Chern-Simons gauge theories have been the subject of great attention during the past decade [1, 2]. In the non-Abelian case, the interest in pure ChernSimons theories stems from their topological character, whereas the Abelian Chern-Simons gauge field is responsible for inducing generalized statistics on matter fields, a phenomenon intrinsic to three-dimensions.

A distinctive feature of Chern-Simons theories is that the requirement of gauge invariance leads to non perturbative effects prior to quantization. In the non-Abelian case, gauge transformations fall into topological classes labeled by $\pi_{3}(G)=\mathbb{Z}$, corresponding to the mapping of $S_{3}$ (compactified three-dimensional space) to the group manifold $G$. Under gauge transformations with non-vanishing winding, the Chern-Simons action $S_{C S}$ is not invariant, and requiring the invariance of $\exp \left(i S_{C S}\right)$ leads to the quantization of the non-Abelian Chern-Simons coefficient [2]. In the Abelian case there is no such topological structure, so the Abelian Chern-Simons coefficient remains arbitrary, and free to induce any desired generalized statistic on the matter fields.

When the theory is formulated at finite temperature, the time direction is effectively compactified into a circle. Since the Abelian group manifold is also a circle, it is tempting to ask whether the Abelian Chern-Simons coefficient remains arbitrary at finite temperature. In this letter we analyze that question, and show that under certain general conditions the Abelian ChernSimons coefficient is indeed quantized at any finite temperature. However, this quantization turns out to be of a different origin and character as compared to the non-Abelian case. It requires the coupling with matter fields, and results from the interplay of the winding of gauge transformations around the compactified time axis, and the winding of the (pure gauge) potential at spatial infinity. The latter determines the total flux of the magnetic field, and in the presence of matter fields specifies the vacuum of the theory. Our result states that when the theory is defined in a vacuum with non vanishing magnetic flux (or electric charge), the requirement of gauge invariance leads at finite temperature to the quantization of the Chern-Simons coefficient and its quantum corrections. Indeed, the situation is similar to that encountered at zero temperature when the theory is formulated in an appropriately compactified spacetime manifold [10, 12]

Our starting point is the action for massive Dirac fermions coupled to an 
Abelian Chern-Simons field at finite temperature

$$
S=S_{F}+i \frac{\theta e^{2}}{4 \pi} S_{C S}
$$

where $S_{C S}$ is the Chern-Simons action

$$
S_{C S}=\int_{0}^{\beta} d \tau \int d^{2} x \epsilon_{\mu \nu \lambda} A_{\mu} \partial_{\nu} A_{\lambda}
$$

and $S_{F}$ is the fermion action

$$
S_{F}=\int d^{3} x \bar{\psi}(\not \partial+i e \not A+m) \psi .
$$

Finite temperature calculations are done as usual, compactifying the (Euclidean) time variable $\tau$ into the range $0 \leq \tau \leq \beta=1 / T$ (in our units, $\hbar=c=k=1)$. Then, the partition function is defined as

$$
\mathcal{Z}=\mathcal{N}(\beta) \int \mathcal{D} \bar{\psi} \mathcal{D} \psi \mathcal{D} A_{\mu} \exp (-S)
$$

where the functional integral must be computed using periodic (antiperiodic) boundary conditions in time for bosons (fermions). It is important to stress that the integration over gauge fields ranges over all periodic configurations compatible with the boundary conditions at spatial infinity to be discussed below. Using the standard Fadeev-Popov procedure, one gets

$$
\mathcal{Z}=\mathcal{N}(\beta) \int \mathcal{D} \bar{\psi} \mathcal{D} \psi \mathcal{D} A_{\mu} \mathcal{D} \chi \delta[F[A]] \Delta_{F P}[A] \exp \left(-S\left[\bar{\psi}, \psi, A^{\chi}\right]\right)
$$

Here $\Delta_{F P}$ is the Fadeev-Popov determinant associated with the gauge-fixing condition $F[A]=0$, and $\mathcal{D} \chi$ is the integration over the group of gauge transformations. The action of these gauge transformations on the fields is given by

$$
\begin{aligned}
A_{\mu}(\tau, x) & \rightarrow A_{\mu}^{\chi}(\tau, x)=A_{\mu}(\tau, x)+\partial_{\mu} \chi(\tau, x) \\
\psi(\tau, x) & \rightarrow \exp [-i e \chi(\tau, x)] \psi(\tau, x) \\
\bar{\psi}(\tau, x) & \rightarrow \exp [+i e \chi(\tau, x)] \bar{\psi}(\tau, x) .
\end{aligned}
$$

In order to preserve the temporal boundary conditions for both bosonic and fermionic fields, the function $\chi$ must satisfy

$$
\begin{aligned}
\partial_{\mu} \chi(\beta, x)-\partial_{\mu} \chi(0, x) & =0 \\
\exp [-i e \chi(\beta, x)]-\exp [-i e \chi(0, x)] & =0 .
\end{aligned}
$$


Let us write the partition function as

$$
\mathcal{Z}=\mathcal{N}(\beta) \int \mathcal{D} A_{\mu} \delta[F[A]] \Delta_{F P}[A] \exp \left(-S_{\text {eff }}[A]\right)
$$

where we have defined the effective action $S_{\text {eff }}[A]$ by

$$
\exp \left(-S_{e f f}[A]\right)=\int \mathcal{D} \bar{\psi} \mathcal{D} \psi \mathcal{D} \chi \exp \left(-S\left[\bar{\psi}, \psi, A^{\chi}\right]\right)
$$

or, after integration over the fermion fields,

$$
\exp \left(-S_{e f f}[A]\right)=\int \mathcal{D} \chi \exp \left(i \frac{\theta e^{2}}{4 \pi} S_{C S}\left[A^{\chi}\right]\right) \times \operatorname{det}\left(\not \partial+i e \not \not^{\chi}+m\right) .
$$

The fermion determinant has been profusely analyzed, both at zero and at finite temperature [3]-[4]. Various perturbative treatments coincide in showing that the determinant has a parity-violating part, to be discussed below, and a parity-conserving piece which contains the Maxwell action. The presence of the latter is important here, since it fixes the boundary conditions for the gauge fields at spatial infinity. In order for a field configuration to have finite energy, the field strength must vanish at infinity, and therefore the gauge potentials must tend to pure gauges.

Evidently, the effective action $S_{\text {eff }}[A]$ defined by eq.(11]) is gauge invariant: $S_{e f f}\left[A^{\eta}\right]=S_{\text {eff }}[A]$ for any $\eta$ satisfying the appropriate boundary conditions. Moreover, if the Chern-Simons action and the fermion determinant were gauge invariant, the integration over the gauge group would factor out, and this effective action would reduce to the usual one obtained after integrating over the fermion fields. Here, however, we shall see that, even in this Abelian theory, there is a gauge dependence due to 'large' gauge transformations. Thus, $S_{\text {eff }}$ will receive a non trivial contribution from the integration over gauge transformations, which precisely restores gauge invariance, even under large gauge transformations.

The set $\mathcal{G}$ of all gauge transformations is partitioned into topological classes $\mathcal{G}_{n}$, classified by an integer $n$ denoting how many times they wind around the time coordinate, according to

$$
\chi^{(n)}(\beta, x)-\chi^{(n)}(0, x)=\frac{2 \pi}{e} n .
$$


A representative of $\mathcal{G}_{n}$ may be taken as a gauge transformation defined by a function $\chi^{(n)}$ of the form

$$
\chi^{(n)}(\tau, x)=\frac{2 \pi}{e} \frac{n \tau}{\beta}+\chi^{(0)}(\tau, x)
$$

where $\chi^{(0)}(\beta, x)=\chi^{(0)}(0, x)$. Such elements obviously satisfy (8) and (12). These transformations have been considered in the case of non-compact $\mathrm{QED}_{3}$ in Ref. [5], where their relevance to the study of confinement was discussed.

The integration over gauge transformations in eq. (11) can be written as a sum over integrations within each topological sector, to get

$$
\exp \left(-S_{e f f}[A]\right)=\sum_{n} \int \mathcal{D} \chi^{(n)} \exp \left(i \frac{\theta e^{2}}{4 \pi} S_{C S}\left[A^{\chi^{(n)}}\right]\right) \times \operatorname{det}\left(\not \partial+i e \not \chi^{\chi^{(n)}}+m\right) .
$$

To proceed further we must determine the way in which the integrand in this expression transforms under 'large' $(n \neq 0)$ gauge transformations. Concerning the Chern-Simons action, from eq. (2) one easily verifies that

$$
S_{C S}\left[A^{\chi^{(n)}}\right]=S_{C S}[A]+\delta_{\chi^{(n)}} S_{C S}[A]
$$

where

$$
\begin{aligned}
\delta_{\chi^{(n)}} S_{C S}[A] & =\int_{M} d^{3} x \epsilon_{\mu \nu \lambda} \partial_{\mu} \chi^{(n)} \partial_{\nu} A_{\lambda}=\int_{M} d^{3} x \partial_{\mu}\left(\epsilon_{\mu \nu \lambda} \chi^{(n)} \partial_{\nu} A_{\lambda}\right) \\
& =\int_{0}^{\beta} d \tau \int d^{2} x\left[\partial_{\tau}\left(\epsilon_{j k} \chi^{(n)} \partial_{j} A_{k}\right)+\partial_{j}\left(\epsilon_{j k} \chi^{(n)} F_{k 0}\right)\right] .
\end{aligned}
$$

The second integral in this expression vanishes due to the boundary condition at infinity discussed earlier for the field strength. For the first term, we have

$$
\begin{aligned}
\int_{0}^{\beta} d \tau \int d^{2} x \partial_{\tau}\left(\epsilon_{j k} \chi^{(n)} \partial_{j} A_{k}\right) & =\int d^{2} x\left[\chi^{(n)}(\beta, x) \epsilon_{j k} \partial_{j} A_{k}(\beta, x)\right. \\
& \left.-\chi^{(n)}(0, x) \epsilon_{j k} \partial_{j} A_{k}(0, x)\right] \\
& =\int d^{2} x\left[\chi^{(n)}(\beta, x)-\chi^{(n)}(0, x)\right] \epsilon_{j k} \partial_{j} A_{k}(0, x) \\
& =\frac{2 \pi n}{e} \int d^{2} x \epsilon_{j k} \partial_{j} A_{k}(0, x) \\
& =\frac{2 \pi n}{e} \Phi(0)
\end{aligned}
$$


where the boundary condition (12) and the periodicity of the gauge fields have been used. Here, $\Phi(\tau)$ denotes the magnetic flux through the spatial plane at 'time' $\tau$ :

$$
\Phi(\tau)=\int d^{2} x \epsilon_{j k} \partial_{j} A_{k}(\tau, x) .
$$

which can be written in terms of the gauge potential at spatial infinity as

$$
\Phi(\tau)=\oint d x^{i} A_{i}(\tau, x)
$$

As discussed earlier, the gauge potential in the integrand of this expression must be a pure gauge. Thus, taking into account the transformation law of the fermion fields in eq. (6), we see that the flux $\Phi$ must be of the form

$$
\Phi(\tau)=\frac{2 \pi}{e} q
$$

where, in general, $q$ is an integer-valued function of $\tau$. However, field configurations with different fluxes at different 'times' $\tau$, will be separated by barriers of infinite energy. Since the Abelian theory has no instantons [6] that may connect those configurations, we must conclude that the value of $\Phi$ in eq. (20) is $\tau$-independent, and consequently, $q$ is a fixed integer, which labels the vacuum of the theory and determines the boundary conditions for the functional integral defining the partition function. In other words, each value of $q$ defines a different theory, in a way analogous to the different vacua of theories with spontaneous symmetry breaking. The partition function corresponding to each of these theories will be denoted by $\mathcal{Z}_{q}$ and, from eq. (9), is given by

$$
\mathcal{Z}_{q}=\mathcal{N}(\beta) \int \mathcal{D} A_{\mu}^{(q)} \delta\left[F\left[A^{(q)}\right]\right] \Delta_{F P}\left[A^{(q)}\right] \exp \left(-S_{\text {eff }}\left[A^{(q)}\right]\right)
$$

where $\mathcal{D} A_{\mu}^{(q)}$ denotes the functional integral measure subject to the boundary condition specified in eq. (20).

The condition on the total flux in the functional integral (21) can be converted into a condition on the total charge $Q=e \int d^{2} x \bar{\psi} \gamma_{0} \psi$, due to the constraint

$$
Q(\tau)=-\frac{\theta e^{2}}{2 \pi} \Phi(\tau)
$$


which follows from (四). Thus, condition (201) implies

$$
Q=-\theta e q
$$

i.e., $\mathcal{Z}_{q}$ in (21) can be equivalently regarded as a partition function in a fixed-charge ensemble. Such partition functions, where conserved internal quantum numbers are given prescribed values, have been considered previously in the literature [7, 8]. The application of this idea to the present case amounts to Fourier transforming the partition function with an imaginary chemical potential (see [8] for details). This may turn out to be a practical procedure to enforce the condition (20) in the functional integral for $\mathcal{Z}_{q}$.

Coming back to the gauge dependence of the Chern-Simons action, we must note that when the theory is quantized with $q \neq 0$, the simple discussion of $\delta_{\chi^{(n)}} S_{C S}$ in eqs. (16-17) is not quite correct. Indeed, in that case the gauge potential $A^{(q)}$ cannot be defined globally without introducing singularities or, alternatively, one must define $A^{(q)}$ resorting to several coordinate patches. As shown in refs. 19, 10], that leads to an extra factor of two in eq. (17), which together with eqs. (15) and (20) lead to

$$
\delta_{\chi^{(n)}} S_{C S}\left[A^{(q)}\right]=\frac{(2 \pi)^{2}}{e^{2}} 2 n q .
$$

We now discuss the way in which the fermion determinant changes under large gauge transformations. An exact expression for the determinant is unknown, but perturbative analysis [4] suggest that its parity-violating part is proportional to the Chern-Simons action, with a temperature dependent coefficient:

$$
\left.\operatorname{det}(\not \partial+i e \not A+m)\right|_{P V}=\exp \left(i \frac{e^{2}}{4 \pi} F(T) S_{C S}[A]\right) .
$$

where the subindex $P V$ denotes the parity-violating contribution. Concerning the known parity-conserving terms, they are gauge invariant even under large gauge transformations, and will play no role in the analysis that follows. Then, assuming that eq. (25) holds, we get

$$
\exp \left(-S_{e f f}\left[A^{(q)}\right]\right)=\sum_{n} \int \mathcal{D} \chi^{(n)} \exp \left(i \frac{e^{2}}{4 \pi}(\theta+F(T)) S_{C S}\left[A^{(q) \chi^{(n)}}\right]\right) .
$$


Then, using eqs. (15) and (24), we have

$$
\begin{aligned}
\exp \left(-S_{\text {eff }}\left[A^{(q)}\right]\right)= & \exp \left(i \frac{e^{2}}{4 \pi}(\theta+F(T)) S_{C S}\left[A^{(q)}\right]\right) \\
& \times \sum_{n} \exp (i 2 \pi(\theta+F(T)) q n),
\end{aligned}
$$

where we have omitted an infinite normalization constant arising from the volume of integration over the gauge group. Thus, we have

$$
\begin{aligned}
& \exp \left(-S_{\text {eff }}\left[A^{(q)}\right]\right)= \\
& \quad \exp \left(i \frac{e^{2}}{4 \pi}(\theta+F(T)) S_{C S}\left[A^{(q)}\right]\right) \sum_{k} \delta((\theta+F(T)) q-k) .
\end{aligned}
$$

Hence, the partition function vanishes, unless

$$
(\theta+F(T)) q=p
$$

where $p$ is an integer-valued function of $T$.

For the theory in a $q \neq 0$ sector, this relation yields a novel quantization rule. Since it must hold even if we start with $\theta=0$, this result states that $F(T)$, the coefficient of the Chern-Simons term induced by the fermionic quantum fluctuations at finite temperature, must be a rational-valued function of the temperature. This, in turn, implies that in the theory at finite temperature, $\theta$ itself must be a rational number. (Actually, the denominators in these rationals is $q$, which is fixed). Notice that our argument does not exclude a possible temperature-dependence of $F(T)$ : it only states that at most it can be a rational-valued function of $T$.

Perturbative analysis leading to a temperature dependence for the fermion determinant of the form (25), make at some point in the calculation a $1 / \mathrm{m}$ expansion, and produce a function $F(T)$ of the form

$$
F(T)=\frac{1}{2} \tanh \left(\frac{m \beta}{2}\right) .
$$

Those perturbative calculations have been performed only in what we have denoted here as the $q=0$ sector, where we obtain no conditions for $\theta$ or $F(T)$. Yet, it is interesting to note that in the limit $m \rightarrow \infty$, where the $1 / m$ 
expansion could be expected to be exact, this expression tends to a step function, up to exponentially small corrections, thus satisfying the quantization condition (29).

It is interesting at this point to compare the situation in the Abelian and the non-Abelian models. For the latter, it is well known that the Chern-Simons coefficient has to be quantized already at zero temperature if $\exp \left(i S_{C S}\right)$ is to be invariat under large gauge transformations [2]. In contrast, no quantization condition arises at $T=0$ for the Abelian theory (unless the spatial dimensions are compactified [10, 12].) For the non-Abelian theory, it was argued in ref. [13] that the Chern-Simons coefficient must remain an integer at any finite temperature. Thus, any temperature-dependent renormalization of the non-Abelian Chern-Simons coefficient must reduce to an integer shift. Here we have seen that an analogous result holds also in the Abelian theory: in any $q \neq 0$ sector, a temperature-dependent renormalization of the Chern-Simons coefficient must satisfy condition (29), so it cannot be a smooth function of the temperature. This will be of importance for the applications of the theory to anyonic superconductivity, where the cancellation of the bare Chern-Simons coefficient against its quantum corrections plays a crucial role [14]. A quantization condition like eq. (29) guarantees that that cancellation will hold at least up to some finite non-zero temperature, thus surviving to low temperature effects. Also, one may expect that as in the non-Abelian case, the quantization of the Chern-Simons coefficient will provide a non-perturbative basis for its non-renormalization to higher orders [15]. This, in turn, is important for the applications of the theory to the Fractional Quantum Hall effect 16.

As opposed to the non-Abelian case, in the Abelian theory the presence of fermions (or scalars, for that matter) is essential for the argument leading to the quantization of the Chern-Simons coefficient at finite temperature. Indeed, it is the presence of matter fields that forces conditions (8) and (12) on the allowed gauge transformations, and determine the behavior of the gauge fields at spatial infinity, leading to the quantization of the magnetic flux by the integer $q$ in eq. (20). As discussed above, different values of $q$ label theories built over different classical vacua, there being no tunneling between the different $q$-sectors. We have shown that in each $q$-theory the Abelian Chern-Simons coefficient is quantized at finite temperature in multiples of $1 / q$, except for $q=0$ where our analysis imposes no conditions on $\theta$ or $F(T)$.

In topological terms, what we have shown is that the quantization of the 
abelian Chern-Simons coefficient at finite temperature, results from the interplay of two different $\pi_{1}(U(1))=\mathbb{Z}$. First, there is the $\pi_{1}(U(1))$ whose elements $q$ label the different classical vacua, and determine the boundary conditions at spatial infinity defining the partiton function $\mathcal{Z}_{q}$ in eq. (21). Then, to enforce the invariance of $\mathcal{Z}_{q}$ under 'large' gauge transformations, we summed over the integers in the $\pi_{1}(U(1))$ which classify the gauge transformations allowed at finite temperature, as specified in eq. (12). As a result, for $T>0$, the coefficient of the Chern-Simons term is quantized according to eq. (29) in each $q \neq 0$ sector. It is interesting to note that these conclusions hold for any $T>0$, and therefore will hold also in the $T \rightarrow 0^{+}$limit.

Acknowledgements. This work was supported in part by FONDECYT, under Grant No. 1950794, by CONICET, under Grant PID 3049/92, the ICTP and by Fundación Andes and Fundación Antorchas. FAS thanks the Pontificia Universidad Católica de Chile for its kind hospitality.

\section{References}

[1] W. Siegel, Nucl. Phys. B156 (1979) 135; J. Schonfeld, Nucl. Phys. B185 (1981) 157.

[2] S. Deser, R. Jackiw and S. Templeton, Phys. Rev. Lett. 48 (1982) 975; Ann. Phys. (N.Y.) 140 (1982) 372.

[3] A.N. Redlich, Phys. Rev. Lett. 52 (1984) 18; Phys. Rev. D29 (1984) 2366.

[4] K. Babu, A. Das and P. Panigrahi, Phys. Rev. D36 (1987) 3725; A. Das and S. Panda, J. Phys. A25, (1992) L245; I.J.R. Aitchinson, C.D. Fosco and J.A. Zuk, Phys. Rev. D48 (1993) 5895.

[5] G. Grignani, G. Semenoff and P. Sodano, 'Confinement-deconfinement phase-transition in 3-dimensional QED', hep-th/9504105, Phys. Rev. D53 (1996), to appear.

[6] R. Pisarski, Phys. Rev. D34 (1986) 3851.

[7] K. Redlich and L. Turko, Z. Phys. C5 (1980) 201. 
[8] J. Kapusta, Nucl. Phys. B196 (1982) 1.

[9] O. Alvarez, Comm. Math. Phys. 100 (1985) 279.

[10] A. P. Polychronakos, Nucl. Phys. B281 (1987) 241.

[11] A. P. Polychronakos, Phys. Lett. B241 (1990) 37.

[12] Y. Hosotani, Phys. Rev. Lett. 62 (1989) 2785; ibid. 64 (1990) 1690.

[13] D. Cabra, E. Fradkin, G.L. Rossini and F.A. Schaposnik, 'Gauge invariance and finite temperature effective actions of Chern-Simons gauge theories with fermions', hep-th/9507136.

[14] E. Fradkin, Phys. Rev. B42 (1988) 570; T. Banks and J. Lykken, Nucl. Phys. B336 (1990) 500.

[15] S. Coleman and B. Hill, Phys. Lett. 159B (1985) 184.

[16] S. C. Zhang, T. Hansson and S. Kivelson, Phys. Rev. Lett. 62 (1989) 82; A. Lopez and E. Fradkin, Phys. Rev. B44 (1991) 5246; X. G. Wen and A. Zee, Phys. Rev. Lett. 69 (1992) 1811. 